\title{
LE KOKANEE, UN SAUMON ADAPTÉ A LA VIE DANS LES EAUX DOUCES
}

\author{
par Bernard BARBIER \\ D. E. A. d'Ecologie, \\ Agent Contractuel du \\ Conseil Supérieur de la Pêche \\ Division: Qualité des Eaux, Pêche \\ et Pisciculture - CERAFER
}

\section{I - INTRODUCTION}

Le saumon est un poisson amphibiatique : dans son cycle normal de vie, il est appelé à fréquenter successivement les eaux douces et les eaux marines. A l'approchie de la maturité sexuelle, il remonte dans son cours d'eau d'origine où il assure sa reproduction : il est potamotoque.

Or, comme la plupart des migrateurs potamotoques dont la croissance s'effectue en mer, il est victime du développement $\in t$ de l'équipement industriel : pollutions et barrages sont autant d'obstacles infranchissables et chaque année plus nombreux, à son retour dans ses aires de fraye.

Si l'on considère les migrations comme des procédés de défense de l'espèce vis-à-vis du milieu environnant, avec pour objet la satisfaction optimale des besoins nutritifs et la réalisation de la reproduction dans les meilleures conditions possibles, on voit alors tout le danger cue représente pour celle-ci l'impossibilité d'èmigrer.

II ne reste plus que l'alternative suivante : ou l'espèce tente de s'adapter à des conditions nouvelles, ou elle disparait de la région. C'est bien cette dernière éventualité qui semble menacer notre saumon atlantique (Salmo salar L.).

Par contre, dans certaines autres régions du globe on a découvert depuis pilus d'un demi-siècle que certaines variétés de saumons se sont naturellement adaptées à une vie holobiotique en eau douce. C'est notamment le cas d'une variété du Pacifique : il s'agit de la forme aland-locked "du saumon Sockeye (Oncorhynchus nerka). de KOKANEE.

Cette forme "Continentale" d'eau douce est plus connue sous le nom Article available at http://www.kmae-journal.org or http://dx.doi.org/10.1051/kmae:1971006 


\section{$1 I$ - NOMS VERNACULAIRES}

La plupart des auteurs désignent généralement par KOKANEE la forme naine, *land-locked s, du saumon Sokeye rouge anadrome : Oncorhynchus nerka kennerlyi SUCLEY (ou encore Oncorhynchus nerka nerka WALBAUM).

Bien que la plupart des biologistes aient adopté ce nom, le kokanee est désigné, suivant les régions par :

$\begin{array}{llll}\text { red fish } & \text { silver } & \text { sockeye } & \text { kennerly's salmon } \\ \text { little red fish } & \text { silver trout } & \text { land-locked sokeye } & \text { kennerly's trout } \\ \text { little red } & \text { silver sides } & \text { land-locked salmon } & \text { kikaninny } \\ \text { red salmon silver salmon land-locked red salmon } & \text { blue back } \\ \text { walla } & \text { land-locked sokeye salmon yank } \\ \text { Les Indiens le nomment "Kokos", les Japonais "Benimasa ", "Khime- } \\ \text { masu "et *KOKAYNEE". }\end{array}$

\section{III - REPARTITION GEOGRAPHIQUE}

Le Kokanee est originaire du Nord-Ouest de l'Amérique du Nord : région allant de l'Orégon à l'Alaska. On l'a trouvé dans les Etats suivants: Orégon, Idaho, Washington, Colombie Britannique et, vers le Nord, Alaska jusqu'à la baie de Bristol, limite Nord-Ouest de la zone côtière.

On l'a trouvé aussi dans l'Est de la Sibérie et dans la péninsule de Kamtchatka. kaido.

Au Japon, il est originaire des lacs Akan et Khemikeppu au nord d'Hok-

On l'a introduit dans l'Etat du Maine, la Californie (1941), le Montana, le Connecticut, l'Etat de New-York, le Colorado, le Yermont, le Dakota du Nord, le Nevada, I'Utah et le Wyoming. Il a été en cutre transplanté dans de nombreux autres lacs et Réservoirs des Etats-Unis ou il se reproduit normalement.

Au Japon, il a été acclimaté avec succès dans de nombreux lacs.

\section{IV - HISTOIRE ET ORIGINE}

i - Rôle des principaux facteurs du milieu dans la disparition du phénomène migratoire.

Il y a eu au début du siècle bien des confusions au sujet de l'identité st du cycle de vie du Kokanee. En 1925, JORDAN signalait la présence de ce petit saumon dans les lacs de montagne, sans toutefois préciser s'il s'agissait d'une forme holobiotique. Le Kokanee passe toute sa vie en eau douce. II a probablement évolué à partir du Sockeye Résiduel issu du Sockeye anadrome : Oncorhynchus nerka.

HENRY et WARD (1932 )ont émis et discuté plusieurs hypothèses au sujet de l'origine des formes land-locked naturelles. Pour eux, si le saumon ne retourne plus à la mer c'est que des obstacles mécaniques (chutes, barrages...) lempêchent de quitter le lac où il séjourne, ou qu'un attrait quelconque, de nature physico-chimiçue (temperature...) ou physiologique (nutrition), le force à y rester. 
L'origine des lacs japonais dans lesquels vit le Kokanee permet de consolider ces hypothèses : ainsi, le lac Akan s'est formè par suite du barrage de la rivière Akan par des matériaux déposés lors des éruptions des volcans Meokan et Oakan.

Bref, des conditions défavorables au type anadrome, telles les barrières naturelles, empèchant la remontée des cours d'eau par les adultes ou l'avalaison des smolts (la présence d'un épilimnion chaud présenterait une barrière à la migration vers l'aval), seraient propices à l'évolution vers une variété holobiotique d'eau douce.

Mais une interprétation purement mécanique ou physique ne suffit pas à expliquer cette conversion à la vie lacustre. En effet. on connait des lacs qui communiquent avec la mer, dont la distance à la mer est relativement faible et qui possèdent des formes Land-locked. Toutefois le facteur thermique semble jouer un rôle important. Les Sockeye migrateurs de Baker Lake descendaient autrefois la Baker River pour aller à la mer (WARD, 1927 - 1930).

Depuis la construction du barrage de Shannon sur le cours de la Baker River, le lac Shannon a bloqué lavalaison des Saumons Sockeye : le barrage $a$, en effet, créé une large étendue d'eau calme dont l'épilimnion, de température plus élevée bloque, une partie de l'année, le Sockeye dans les zones profondes restees plus froides. Simultanément, la disparition du courant au sein du lac l'incite à hésiter puis à abandonner sa migration et c'est peut-être là le facteur ie plus important.

Des auteurs ont souligné le fait que l'abondance de la nourriture a abouti à la formation des formes land-locked dans certains lacs. Mais là encore, on connait des lacs très riches en nourriture qui possèdent des Sockeye migrateurs.

Il est généralement admis actuellement que le Kokanee a évolué à partir du Sockeye anadrome lorsque des modifications de l'environnement ont crée des conditions défavorables à la migration.

Apparamment il n'y a pas de différences structurales entre anadrome et sédentaire. FOERSTER (1947) a relaché des jeunes Kokanee d'un an après les avoir marqués par ablation des nageoires pelviennes et de telle façon qu'ils retournent à la mer. Quatre ans plus tard, vingt-cing de ces poissons âgés de cinq ans, furent récupérés par les pêcheurs professionnels. Leur taille était de l'ordre de celle des Sockeye anadromes de cinq ans de la même rivière. Cette véritable reconversion du Kokanee en anadrome montre donc bien la parenté entre le Sockeye anadrome et le Kokanee, mais il n'est pas certain que cela soit valable pour toutes les races de Kokanee.

Cependant, il semble bien que les différences de taille entre le Sockeye et le Kokanee soient dues plus à l'environnement qu'à l'héréditè.

Par contre VERNON (1957) a publié une étude morphologique détaillée du Kokanee de Kootenay Lake (Sud-Est de la Colombie Britannique) montrant l'existence de trois races dont les caractères seraient en partie háréditaires.

Ce lac comporte 3 bras principaux (fig. 1): 


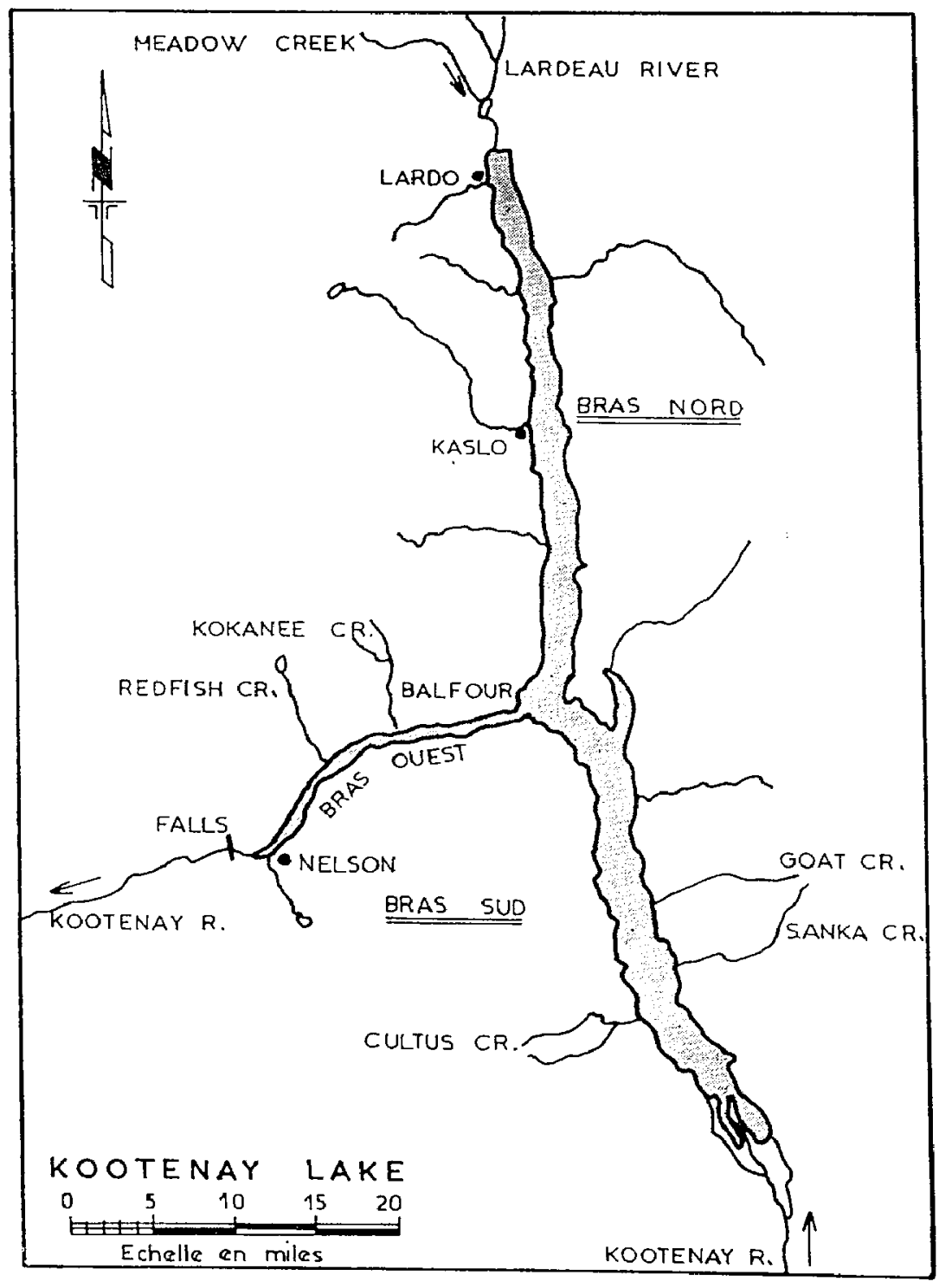

Fig. 1 - Kootenay Lake (Vernon 1957) 
- Bras Sud recevant : Kootenay River,

Goat Creek,

Sanka Creek,

Cultus Creek.

- Bras Nord recevant Meadow Creek, entre autres : Lardeau River.

- Bras Ouest recevant : Redfish Creek,

Kokanee Creek.

Une chute naturelie récente (époque historique) constitue un barrage infranchissable pour les poissons anadromes à l'extrémité du bras Ouest.

L'étude morphologique a montré l'individualisation à l'intérieur de chacune des trois parties du lac et de leurs cours d'eau représentatifs, de trois races distinctes. Les descendants de ces trois races, placés dans des conditions analogues d'environnement, héritent des caractères des géniteurs quant au nombre de vertèbres.

II semble donc dans ce cas que la ségrégation au moment de la reproduction (moins de $3 \%$ d'intrus sur les frayères) due à la forte attraction vers les aires natales, soit le principal facteur de sélection, conduisant à des divergences génotypiques et à une considérable variabilité phénotypique. On pense d'ailleurs que chaque population de Kokanee a évolué à partir d'un groupement spécifique de Sockeye anadrome propre à chaque rivière.

\section{2 - Le "Résiduel" de Cultus Lake.}

Les études faites par RICKER (1938) sur le Cultus Lake, Sud-Ouest de ia Colombie Britannique, paraissent intéressantes quant au comportement des populations du Sockeye anadrome (Oncorhynchus nerka kennerlyi SUCKLEY) de ce lac et nous permettent de mieux comprendre les étapes qui ont pu marquer l'évolution vers le type Kokanee. Le Saumon Sockeye se présente dans Cultus Lake sous trois formes :

1 - Le Sockeye anadrome, normal qui passe par la phase thalassotrophe.

2 - Le groupe des a Résiduels", non anadromes, progéniture du Sockeye anadrome, qui a perdu cette phase thalassotrophe.

3 - Le Kokanee non anadrome, qui n'a pas de rapport avec le Sockeye anadrome et qui passe lui aussi toute sa vie dans le lac.

L'anadrome et le Sockeye a résiduel » représentent deux modes de vie adoptés par les différents membres d'une même population. Le caractère le plus marquant du aRésiduel • semble être la prédominance des mâles dans sa population.

II est fort probable que la prédominance des mâles chez le Sockeye Résiduel s'explique par une ségrégation des sexes au moment où les anadromes descendent vers la mer. Ceci concorde d'ailleurs avec les observations de FOERSTER (1936) qui a montré la prédominance des femelles dans le groupe anadrome de Cultus Lake. Mais dans ce groupe, le "sexe ratio " bien que très variable n'est pas aussi déséquilibré que chez les Résiduels : la proportion des femelles n'a jamais dépassé 0,75 . Il est donc bien clair que les proportions anormales des sexes chez les Résiduels et les Anadromes ne sont pas directement complèmentaires, c'est-à-dire la simple conséquence d'une séparation. 
Le tableau | (RICKER 1938) montre les différentes scissions qui s'opèrent parmi la descendance du Sockeye anadrome. Dans le courant de la deuxième année on peut distinguer 3 groupes de taille : $a, b$ et $c$. Le groupe de grande taille (a) parvient à maturité à l'automne de sa deuxième année en temps que Résiduels de 2 ans (d) ou à l'automne de l'une des 2 années suivantes comme Résiduels de 3 (i) ou de 4 ans (1).

Les grands Résiduels de 4 ans sont rares. Le groupe (b) donne des smolts d'un an qui gagnent la mer. Le groupe (c) renfermant des individus de petite taille subira une scission l'année suivante (3è année), donnant une branche $(e+f)$ de Résiduels de 3 et 4 ans et des smolts de 2 ans (g) qui vont gagner la mer.

Les smolts de 3 ans $(k)$ sont très rares : cette catégorie est basée sur la capture d'un seul spécimen mâle $(21,6 \mathrm{~cm}$ à la capture). Cependant, ils se rencontrent plus souvent dans le Nord de la Colombie Britannique.

Le groupe (a) est constitué presque exclusivement de mâles. Les smolts d'un an (b) ont parfois un excès significatif de femelles. Les Résiduels de 3 ans du groupe (j) sont essentiellement mâles : la plus forte proportion de femelles observée étant 0,066 (ponte de 1933).

La plus forte proportion de femelles dans les Résiduels de 4 ans du groupe $(\mathrm{m})$ a été de 0,3 pour la ponte de 1932. Les smolts de 2 ans (g) étudiés par FOESTER ont un "sexe ratio " normal.

On peut remarquer que, dans les ségrégations successives, ce sont généralement les poissons de plus grande taille qui restent sur place, comme si, après être parvenus à un certain stade de leur croissance, ils n'éprouvaient plus le besoin d'émigrer. Ceci ètant valable surtout pour les mâles.

La présence du Sockeye *Résiduel dans Cultus Lake semble bien représenter une phase intermédiaire de l'évolution du Sockeye anadrome vers le type "land-locked *.

En effet, if résulte des études de RICKER que le type * Résiduel * présente l'ébauche d'une divergence analogue à celle qui a donné le rameau "land-locked".

Dès 1929, alors que l'on procédait à des expériences d'élevage de jeunes Sockeye on fut conduit, tout à fait par hasard, à la production de poissons Résiduels, *land-locked": les lacs CRAWFORD et RAINBOW au Sud de Stuart Lake (latitude 54035) en Colombie Britannique servaient de bassins naturels pour l'élevage des jeunes Sockeye. Quelques années après, on dut supprimer de ces lacs 203 poissons devenus "land-locked "afin de redonner à ces lacs leur fonction originelie: l'élevage des jeunes saumons Sockeye anadromes.

\section{3 - Autres formes *land-locked».}

Quelques autres espèces de saumons présentent des formes land-locked. Ce sont principalement les suivantes:

\section{a) LAND-LOCKED KING SALMON (Oncorhynchus tshawytscha).}

Cette espèce donne peu de saumoneaux land-locked. On n'a jamais pu établir le maintien des populations land-locked, même quand il se présentait des frayères convenables. Les descendants sont plus petits et moins fertiles (Nouvelle-Zélande). 
Dans le New Hampshire, la ponte s'effectue en lac, les produits sexuels sont fertiles et déposés dans des nids creusés par les poissons qui émigrent dans les affluents après la fraie.

La maturité a lieu à 4 ans comme pour la forme anadrome.

Cette forme land-locked est parfois utilisée pour limiter des espèces qui prolifèrent trop.

\section{b) LAND-LOCKED SILVER SALMON (Oncorhynchus kisutch).}

Cette forme peut grandir dans les eaux continentales mais la fraie n'a pas tieu : les piscicultures doivent s'efforcer de maintenir les populations par des implantations régulières. On note une perte de l'instinct de reproduction.

\section{c) LAND-LOCKED ATLANTIC SALMON (Salmon salar).}

Dès la fin du siècle passé, on avait noté des formes land-locked de Salmo salar : Maine, Québec et quelques autres provinces maritimes et en Europe dans des lacs de Norvège, de Suède, de Finlande et de Russie.

VIBERT (1947) signale dans le bassin de l'Adour l'existence de jeunes saumons parvenus à maturité sans avoir quitté leur rivière.

Des essais d'introduction, plus ou moins fructueux, ont été faits dans différents Etats américains. Au Canada, dans la province du Québec, LEGENDRE considère ce Saumon a dulcicole» comme le poisson de sport de l'avenir.

\section{$V$ - HABITAT}

Les exigences du Kokanee, poisson pélagique de lac, sont très analogues à celles de la truite de lac. La température optimale se situe aux environs de $10 \circ \mathrm{C}$.

Au-dessus de $15,5 \circ \mathrm{C}$, on enregistre déjà un début de mortalité, au moins chez les jeunes. DILL (août 1947) a établi * qu'un lac convenable pour le Kokanee est profond, possède une zone côtière et un stratification thermique. Dans de tels lacs, le Kokanee se tient dans une zone de $3,30 \mathrm{~m}$ d'épaisseur, dont la température ne dépasse pas $10 \circ \mathrm{C}$.

Un tel comportement vis-à-vis de la température peut expliquer les observations des pêcheurs de Californie qui, au début du printemps et à la fin de l'automne, lorsque les températures superficielles sont faibles, peuvent capturer facilement le Kokanee qui est alors dans la zone supérieure. En été, if se refugie dans l'hypolimnion où les pêcheurs doivent le rechercher.

Un exemple de ce thermopréférendum pour une strate déterminée est donné par le Kokanee de Pine Flat Reservoir en 1960. Pendant la période de stratification thermique, on a tiré de l'eau à un niveau situé à $20 \mathrm{~m}$ en-dessous de la surface; les Kokanee ont alors quitté le lac et on les a retrouvés à une quinzaine de kilomètres en aval.

Le lac Almanor, Plumas Conty, est un grand réservoir cui présente une stratification thermique et chimique durant les mois d'été. Un grand nombre de Kokanee furent récupérés morts ou mourant pendant l'été 1960. La température de surface, pendant les trois premiers jours du mois d'août se situait entre $19 \circ$ et $22^{\circ} \mathrm{C}$. Les poissons s'étaient concentrés clans une zone de soüce sous-lacustre dont la température était de $7,5 \circ \mathrm{C}$; mais cette eau, bien qu'assez froide pour le Kokanee, n'était pas assez riche en oxygène dissous. 
Outre une stratification thermique satisfaisante, le Kokanee exige des eaux suffisamment froides et oxygénées pendant les mois critiques de l'été. Les lacs et réservoirs qui présentent des stratifications chimique et thermique, sans cette couche froide et oxygénée, ne conviennent pas au Kokanee.

Au point de vue trophique l'abondance de zooplancton dans la zone pélagique constitue un élément favorable à une croissance rapide.

Au Japon, le Kokanee s'est admirablement acclimaté dans des lacs d'une profondeur variant de 22 à $363 \mathrm{~m}$, qui présentent une stratification thermique verticale en été $\left(23^{\circ} \mathrm{C}\right.$ en surface et température constante au fond de 4 à $5 \circ \mathrm{\circ}$ ), de bonnes conditions d'oxygénation et de $\mathrm{pH}$, ainsi qu'une abondance de crustacés zooplanctoniques, sa nourriture de base.

\section{VI - CROISSANCE ET NUTRITION}

\section{1 - Le Kokanee, poisson pélagique consommateur de zooplancton.}

Les œufs de Sockeye, pondus en hiver dans les petits affluents des lacs, éclosent au printemps. C'est vers mai-juin que les saumoneaux regagnent le lac pour y mener une existence de poisson pélagique consommateur de plancton. Au cours de leur première année de vie lacustre, ils présentent à l'intérieur des différents bassins des lacs Babine et Nilkitkwa (JOHNSON 1961) une distribution non uniforme (fig. 3).

On a subdivisé ces 2 lacs $\left(475 \mathrm{~km}^{2}\right)$ en 7 bassins. Les densités des populations de jeunes Sockeye sont indiquées pour chaque bassin, en nombre de poissons par hectare. Elles sont fonction essentieliement du nombre et de la longueur des affluents par rapport à la surface de lac correspondante.

Grâce à des filets et des prospections au sonar on a pu mettre en évidence une répartition horizontale typique : pendant le jour les Sockeye se tiennent à une profondeur de 0 à $5 \mathrm{~m}$, avec un maximum de concentration vers $3 \mathrm{~m}$ (fig. $2 \mathrm{~A}$ ). Puis dès la tombée de la nuit, on constate une remontée massive des jeunes vers la surface.

L'existence d'un vent dominant crée parfois un courant au niveau de l'èpilimnion qui entraine une concentration du poisson et du zooplancton vers la rive opposée à ce vent (fig. $2 \mathrm{~B}$ ).

2 - Rythme de croissance de Oncorhynchus nerka relativement à la compétition intraspécifique et à l'abondance de nourriture.

Au cours de sa première année, c'est dans ses premiers mois de vie lacustre, c'est-à-dire pendant la période de stratification thermique de l'été, que la croissance du jeune saumon sera la plus forte. Ainsi un poisson de $0,2 \mathrm{~g}$ à la mi-juin pèsera $4,5 \mathrm{~g}$ à la mi-octobre et seulement $5,5 \mathrm{~g}$ au printemps suivant.

Les relevés faits dans les différents bassins de Babine et Nilkitkwa Lakes fournissent une base de comparaison pour les rythmes de croissance de mi-juin à mi-octobre (tableau II). 


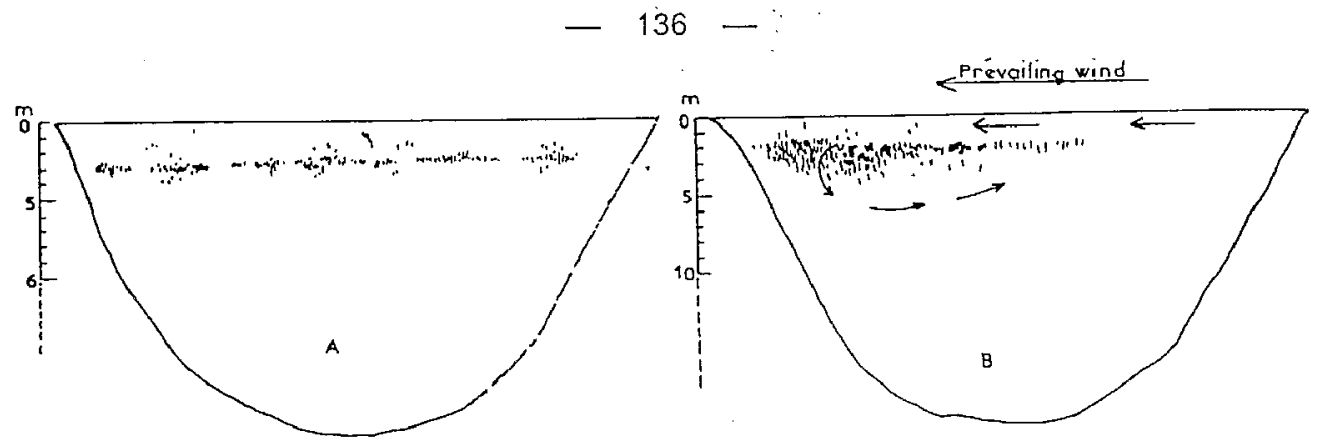

Fig. 2 - Distribution des jeunes poissons d'après les investigations au * sonar n. A) Distribution verticale typique de $O$. nerka (âge $0+$ ) le jour.

B) Effet apparent de la circulation de l'eau sur la distribution horizontale de $O$. nerka (âge 0 ), lors d'un léger vent dominant (19 juillet 1957). (D'après JHONSON 1961).

La localisation des points correspond à l'emplacement des O. nerka.

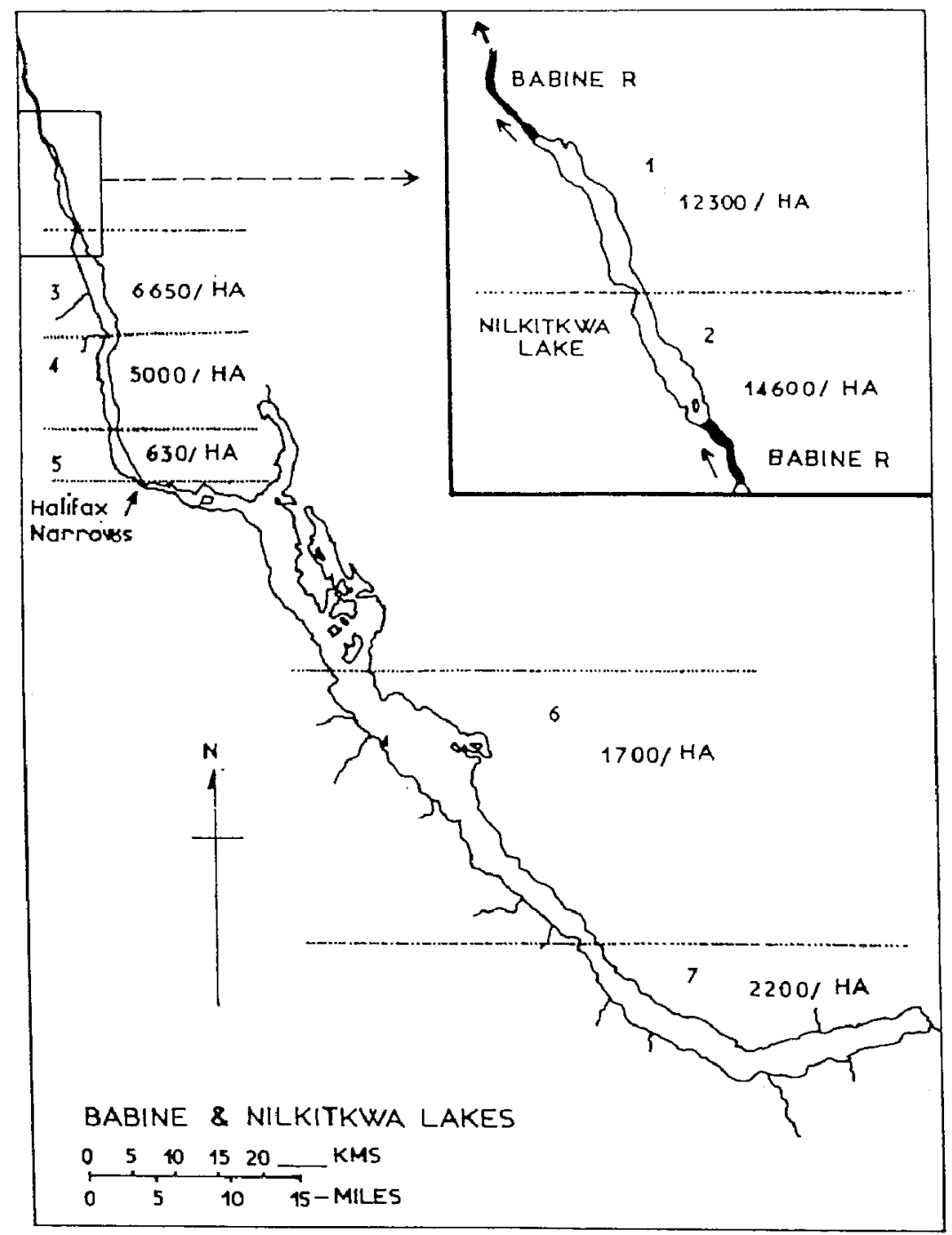

Fig. 3 - Disposition des 7 bassins des Babine et Nilkitkwa. Lakes. Les densités de population de O. nerka (âge $0+$ ) à la fin d'août 1957 sont données, en poissons par hectare, pour chacun des 7 bassins. (D'après JOHNSON 1961). 


\begin{tabular}{|c|c|c|c|c|c|}
\hline Bassin & Année & $\begin{array}{c}\text { Moyenne } \\
\text { des poids } \\
\text { mi-octobre }\end{array}$ & $\begin{array}{c}\text { Densité de } \\
\text { population } \\
\text { fin août }\end{array}$ & $\begin{array}{c}\text { Moyenne de } \\
\text { zooplancton } \\
\text { récolté }(0-5 \mathrm{~m} \text { ) } \\
\text { de mi-juin } \\
\text { à mi-octobre }\end{array}$ & $\begin{array}{c}\text { Moyenne } \\
\text { de } \mathrm{t} \text { oC } \\
\text { de mi-juin } \\
\text { à mi-octobre }\end{array}$ \\
\hline & & $\mathrm{g}$ & Poisson/ha. & mg/m & oC \\
\hline et 2 & 1956 & 3,8 & 3000 & 17 & 13,4 \\
1 & 1957 & 2,2 & 12000 & 12 & 12,0 \\
2 & 1957 & 1,7 & 14000 & 11 & 12,0 \\
3 & 1957 & 3,6 & 6500 & 36 & 13,0 \\
4 & 1957 & 3,8 & 5000 & 69 & 13,4 \\
5 & 1957 & 5,7 & 630 & 80 & 13,4 \\
1 & 1958 & 2,0 & 9900 & 8 & 11,9 \\
2 & 1958 & 1,2 & 16900 & 8 & 11,9 \\
3 & 1958 & 2,2 & 9300 & 21 & 12,3 \\
4 & 1958 & 2,5 & 9050 & 34 & 13,1 \\
5 & 1958 & 3,1 & 7550 & 42 & 13,4 \\
6 & 1958 & 4,2 & 1700 & 102 & 14,4 \\
7 & 1958 & 4,2 & 2250 & 118 & 14,9 \\
3 & 1956 & 4,0 & 120 & 54 & 14,2 \\
7 & 1956 & 5,0 & 350 & 110 & 14,8 \\
6 & 1957 & 5,0 & 1700 & 95 & 12,2 \\
7 & 1957 & 5,0 & 2200 & 120 & 12,6 \\
\hline
\end{tabular}

TABLEAU \| : Relevés effectués dans les différents bassins de Babine et Nilkitkwa Lakes

(D'après JOHNSON, 1961)

Entre juin et octobre le rythme de croissance est fortement influencé par la compétition intraspécifique et l'abondance de la nourriture. JOHNSON considère le poids, en gramme, à la mi-octobre, comme mesure de rythme de croissance, la densité de population à la fin d'août comme mesure de la compétition intraspécifique et la moyenne de zooplancton récolté (poids $\mathrm{sec}$ en $\mathrm{mg}$ par $\mathrm{m}^{3}$ ) comme mesure de l'abondance de nourriture.

C'est à partir des courbes de récolte (de 0 ì $5 \mathrm{~m}$ ) établies pour chaque bassin que les moyennes de plancton récolté furent calculées. relations :

Les graphiques suivants (échelles logarithmiques) représentent les

- Fig. 4A; entre la croissance, de juir à octobre, et l'abondance moyenne de plancton : la croissance augmente avec une augmentation de zooplancton.

- Fig. 4 B; entre la taille moyenne et la densité de population. La corrélation apparait dès que la densité de population dépasse 5000 poissons par hectare. Il y a une diminution progressive de la croissance dès que la densité dépasse ce niveau.

- Fig. $4 \mathrm{C}$; entre la densité de population et l'abondance de zooplancton. La ressemblance avec la fig. $4 \mathrm{~B}$ est nette (sauf pour le point encerclé); on note une réduction progressive de l'abondance du zooplancton par accroissement des densités de populations au-delà d'environ 5000 poissons par hectare. 


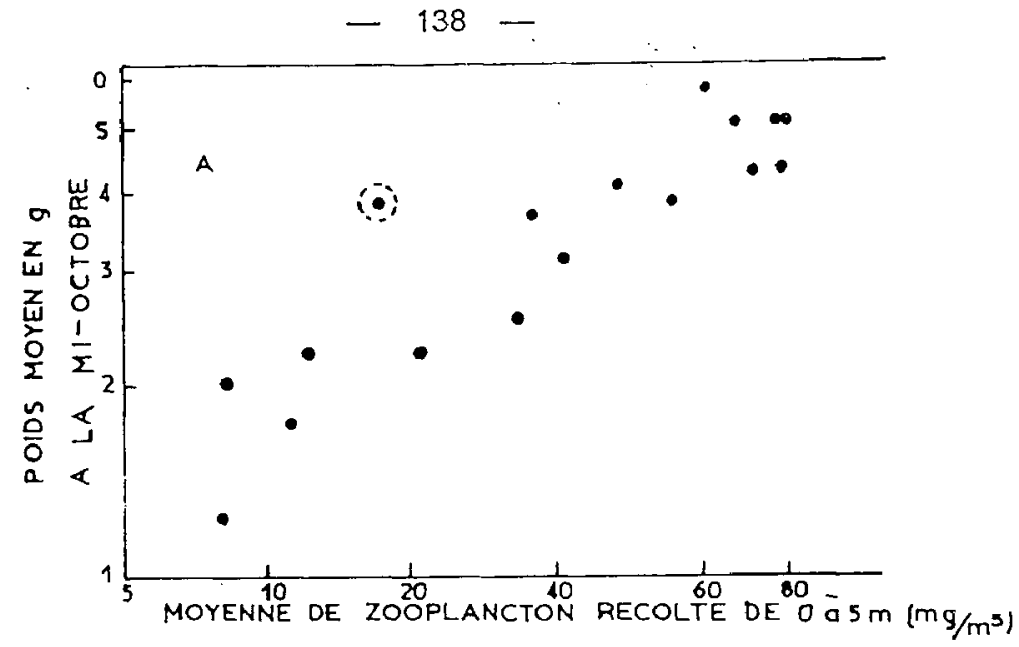

Fig. 4 A. - Relations entre le poids moyen de O. nerka (âge $0+$ ) au milieu d'octobre et la moyenne de zooplancton récolté, de 0 à $5 \mathrm{~m}$, de mi-juin à mi-octobre.

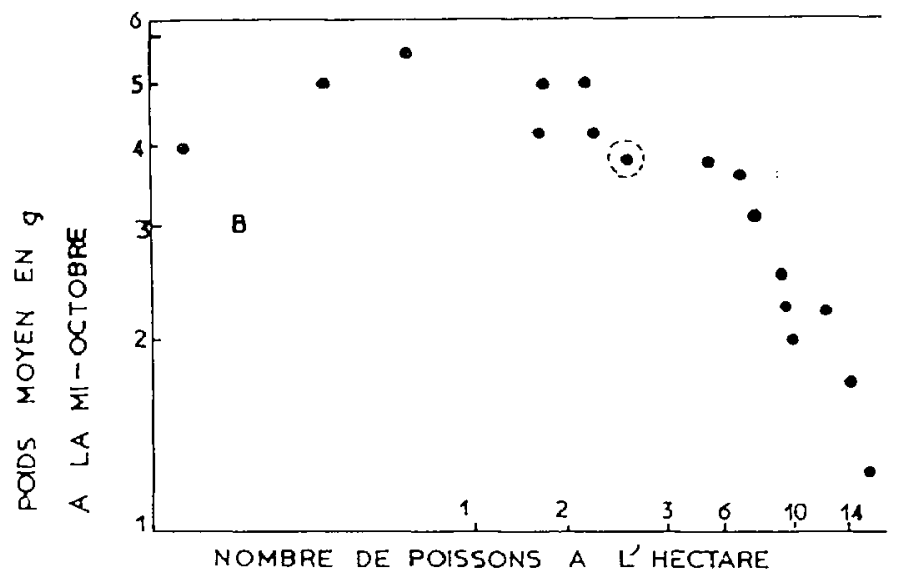

Fig. 4B. - Relations entre le poids moyen de O. nerka (âge 0 t) à la mioctobre et la densité de population de 0 . nerka (âge $0+$ ) à la fin août.

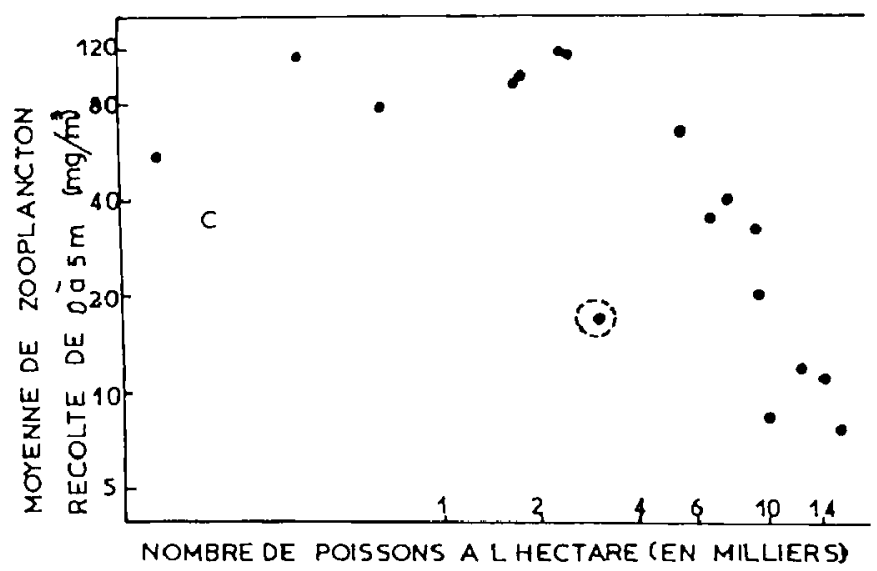

Fig. $4 \mathrm{C}$. - Relations entre la moyenne de zooplancton récolté de 0 à $5 \mathrm{~m}$ de mi-juin à mi-octobre et la densité de population de $O$. nerka (âge 0) à la fin août. (D'après IOHNSON 1961) 
Quand la densité de population augmente au-delà d'un certain niveau critique (la consommation est égale à la production), on observe alors une réduction croissante de la production de nourriture entraînant elle-même une ximinution du rythme de croissance du consommateur.

Dans le lac Pend Oreille les rythmes de croissance au cours des années Euccessives se traduisent par les tailles suivantes :

$$
\begin{aligned}
& 5 \text { à } 7 \mathrm{~cm} \text { pour la } 1 \text { re année. } \\
& \text { - } 18 \text { à } 20 \mathrm{~cm} \text { pour la } 2^{\text {me }} \text { année. } \\
& \text { - } 20 \text { à } 23 \mathrm{~cm} \text { pour la } 3^{\mathrm{me}} \text { année. } \\
& \text { - } 25 \text { à } 30 \mathrm{~cm} \text { pour la } 4 \mathrm{me} \text { année. }
\end{aligned}
$$

Là aussi, c'est de juillet à octobre que s'effectue la plus forte croissance. Elle correspond à la période d'abondance de zooplancton. La croissance est très réduite en hiver.

\section{3 - Composition de la nourriture de l'adulte (analyses stomacales).}

La nourriture du Kokanee est essentiellemerlt constituée de crustacés planctoniques. Les genres dominants sont : Daphnia, Cyclops et Diaptomus.

Des biologistes travaillant sur le Skaguay Reservoir au Colorado ont montré que la nourriture du Kokanee était presque exclusivement constituée par des Entomostracés et quelques imagos d'insectes. Les Cladocères, présents Jans $94 \%$ des estomacs, formaient jusqu'à $88 \%$ de la nourriture.

Les Copépodes, présents dans $57 \%$ des spécimens étudiés arrivaient a former $10 \%$ de la nourriture totale, alors que les Diptères, trouvés dans $20 \%$ des estomacs, constituaient les $2 \%$ restant.

Les études faites sur ce réservoir ont révélé que les Hyménoptères constituaient la plus grande partie en volume de la nourriture de la truite. Mais on ne trouva pas d'Hyménoptères dans l'estomac des Kokanee pris les rnêmes jours.

Le Volvox est la nourriture dont le Kokanee dispose au début du printemps, mais elle n'a pu être mise en évidence après la multiplication des Entomostacés.

Dans l'Idaho on a montré que le Kokanee consommait surtout des Copépodes et des Cladocères.

RICKER (1938) montra que les * Résiduels de Cultus Lake consommaient surtout les crustacés du plancton pélagique, notamment Daphnia et Epischura ainsi que quelques insectes aquatiques et terrestres. II signale en outre que certains grands Résiduels de 4 ans peuvent prélever de jeunes alevins de Cottus asper et même parfois de Sockeye.

NORTHCOTE et LORZ (1966) ont fait des analyses stomacales sur des Kokanee pris au filet dans Nicola Lake, du printemps à l'automne des années i959 à 1963, dans le but d'étudier les variations saisonnières et nycthémérales de la nourriture du Kokanee adulte de Nicola Lake (Colombie Britannique). Les captures furent faites aux stations $\mathrm{S} 1$ et $\mathrm{S} 2$ (fig. 5). La plupart des poissons étaient des reproducteurs de 3 ans, quelques-uns de 4 ans et en octobre 1959 certains étaient dans leur $2^{\mathrm{me}}$ année. Les distributions d'âges et de tailles des Kokanee de Nicola Lake sont données en fig. 6. 


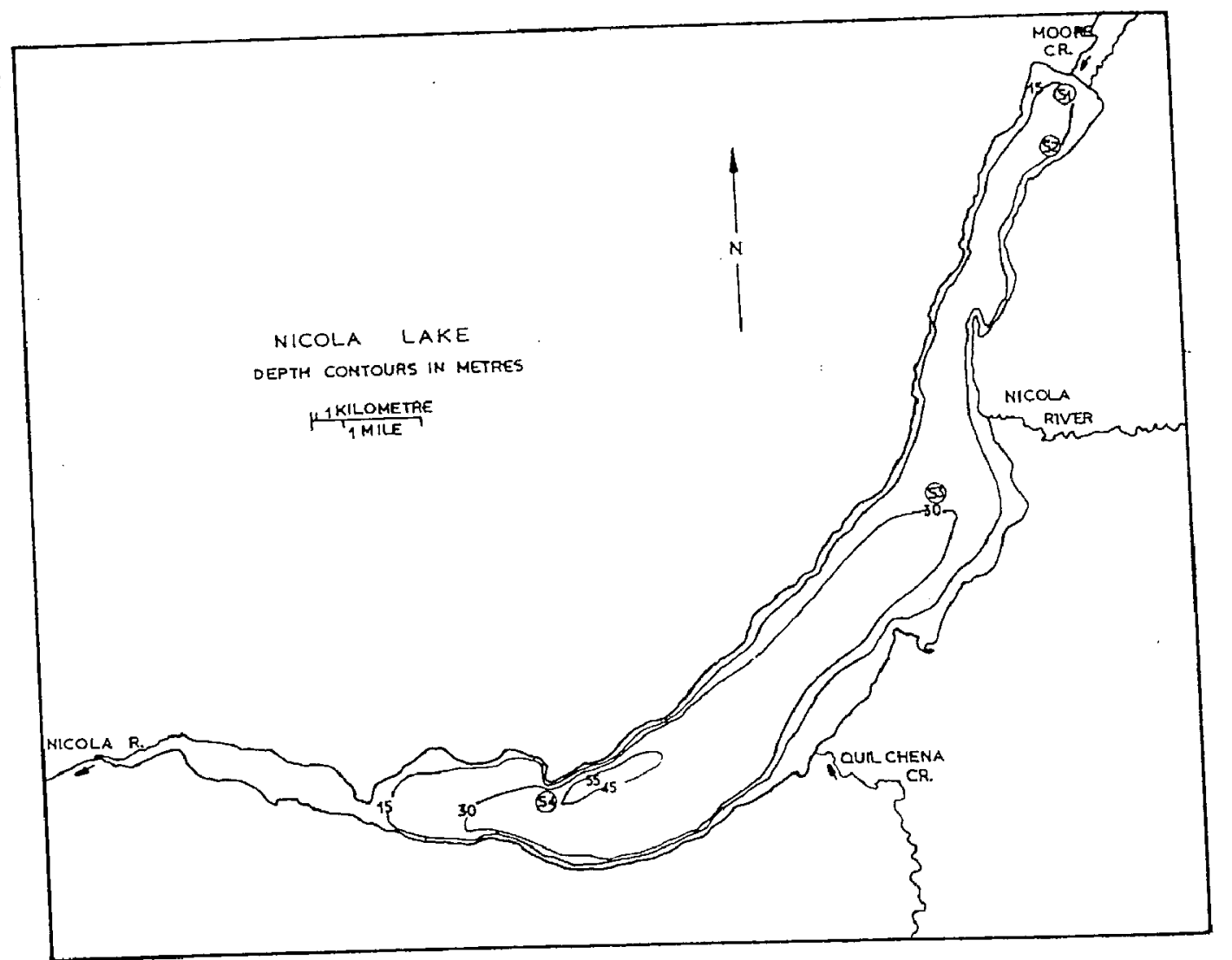

Fig. 5 - Nicola Lake - Les stations de pêche au filet sont notées de S 1 à $S 4$. 

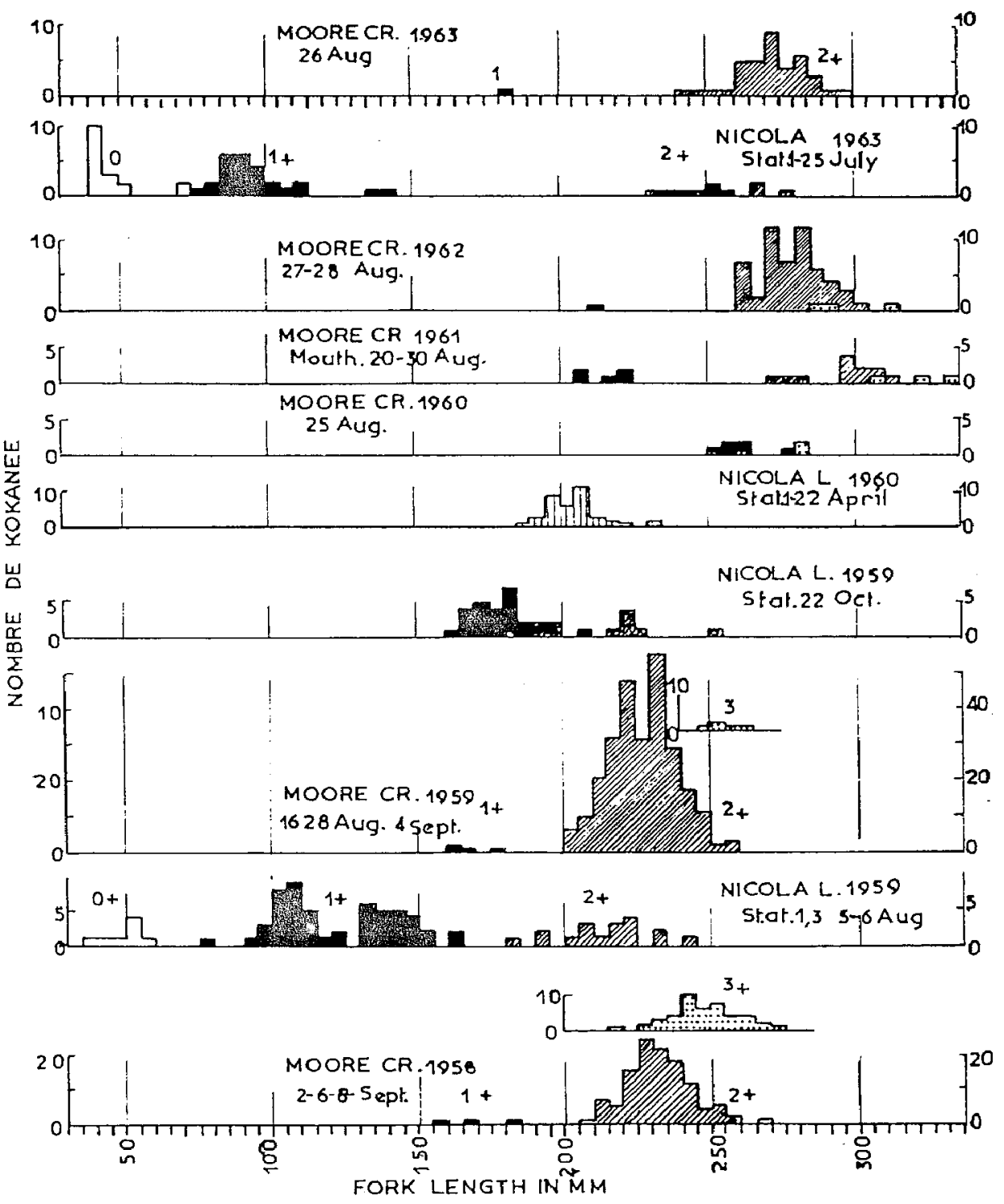

Fig. 6 - Fréquences d'âges et de longueurs des Kokanee de Nicola Lake. (D'après LORZ et NORTHCOTE 1965). 
Après conservation dans une solution à $10 \%$ de formol, le contenu de chaque estomac est enlevé et égoutté. On en détermine le volume. Les restes des éléments constituant la nourriture sont séparés au microscope binoculaire par groupes principaux. On estime alors la proportion de chaque groupe par rapport au volume total de la nourriture.

C'est en juin et en octobre que le volume moyen de la nourriture dans les estomacs des Kokanee est le plus grand, alors qu'il reste faible pour l'été.

Une grande partie de la nourriture consommée au printemps et à l'automne est constituée de crustacés-planctoniques. Les Diaptomides (Sous-cl. Copépodes-O. Gymnoplea) avec surtout Epischura nevadensis et quelques Diaptomus sp. prédominent au printemps alors que les Daphnia (Sous-cl. Branchiopodes, groupe des Cladocères) sont plus importantes en automne. Seul Leptodora kindtii (Branchiopodes, groupe des Cladocères) contribue de façon appréciable à la nourriture du Kokanee adulte en plein été. Mais ce sont surtout les nymphes de Chironomides qui constituent la majeure partie de la nourriture du Kokanee au cours de l'été : $70 \%$ du volume moyen en juin et en juillet (fig. 7).

Les formes dominantes de nymphes sont constituées par Chironomus sp. et Procladius sp.; Cryptocladopelma sp. est moins courant.

Les larves de Chironomides constituent une petite partie de la nourriture de l'été. En outre, les estomacs contiennent quelques particules de zooplancton, d'insectes terrestres et d'acariens qui représentent toujours moins de $10 \%$ du volume.

Des précisions sur les variations nycthémérales des prises de nourriture purent être données : des filets posés et relevés à intervalles réguliers au cours de la journée et de la nuit fournirent les échantillons nécessaires.

On trouva plus de Diaptomides et de Leptodora dans les estomacs des Kokanee capturés de jour. Pour ce qui est des Chironomides, ce sont les larves qui sont surtout consommées le jour alors que les nymphes le sont essentiellement pendant la nuit (fig. 8).

Ce n'est qu'à partir du mois d'août que le volume moyen de nourriture dans les estomacs des Kokanee devint plus faible la nuit que le jour. De plus dans les séries des 24 et 25 aoút 1959 où les échantillons de nuit étaient civisés en 3 périodes, on a pu montrer une diminution progressive du volume moyen de nourriture au cours de la nuit. C'est ce que confirment également les séries nycthémérales des 25 et 26 août 1963 (fig. 9).

On a pu noter par ailleurs une diminution dans le volume moyen de nourriture pendant l'été : ceci semble en relation avec l'approche de la maturité sexuelle, peu avant la migration dans les cours d'eau de fraie, généralement at! milieu d'août.

En effet PLATTS (1958) note lui aussi une diminution, en octobre, de la consommation de nourriture du Kokanee proche de sa maturité, dans le lac Pend Oreille (ldaho), avant le début de la fraie (de novembre à décembre). Dans Nicola Lake, des Kokanee immatures pris en octobre et qui pondront pour la plupart l'automne suivant avaient un volume moyen de nourriture dans l'estomac nettement plus élevé que celui des Kokanee qui arrivaient à maturité cette année-là et qui furent pris un mois avant.

De nombreuses ètudes (CLEMENS, 1939; PLATTS, 1958 ; CHAPMAN et FORTUNE, 1963; HORAK et TANNER, 1964) ont souligné l'importance des 

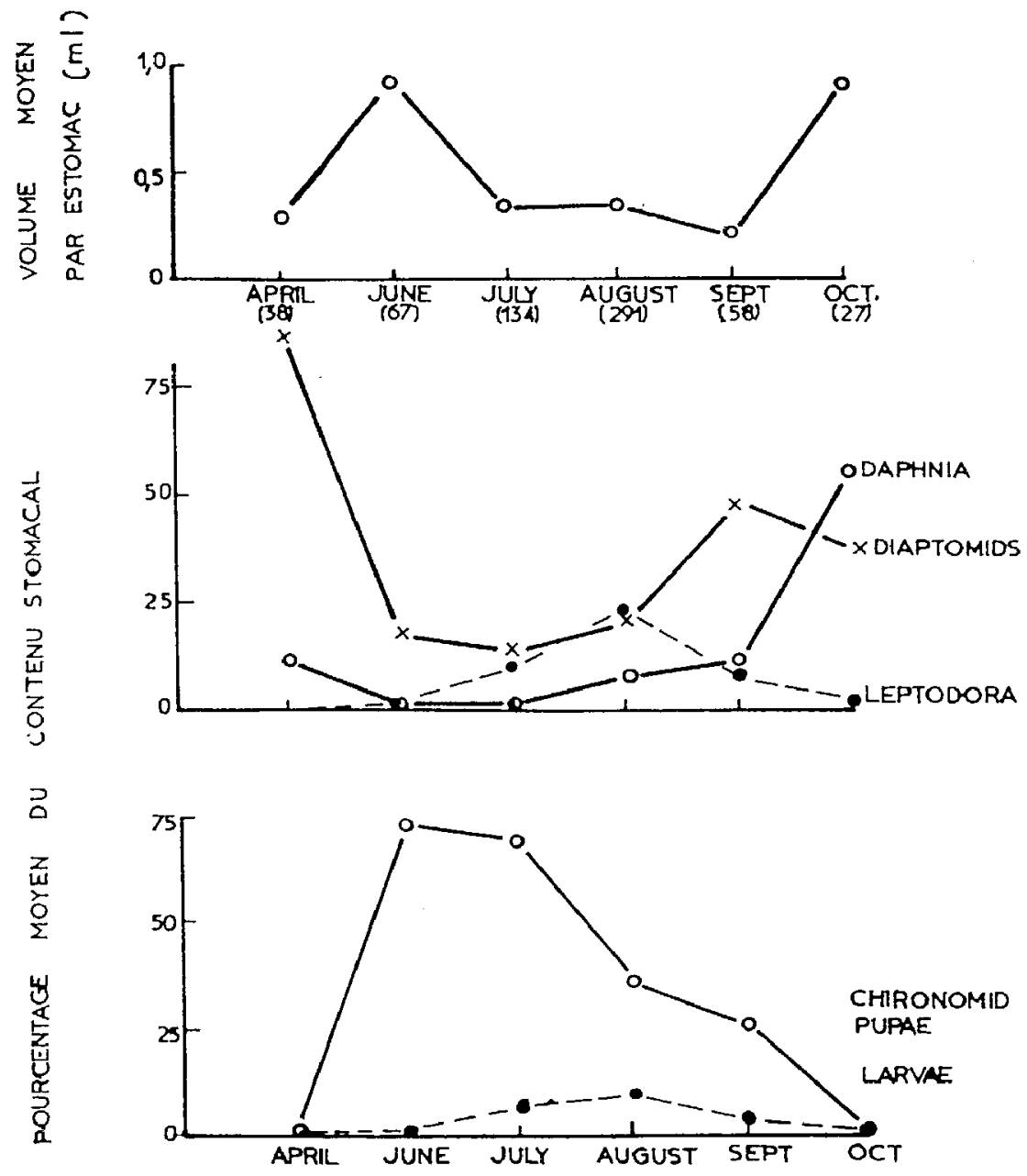

Fig. 7 - Changements saisonniers du volume et de la composition de la nourriture consommée par le Kokanee de Nicola Lake de juin à octobre 1959 et avril 1960.

Le nombre des poissons échantillonnés est indiqué entre parenthèses. (D'après NORTHCOTE et LORZ 1966) 


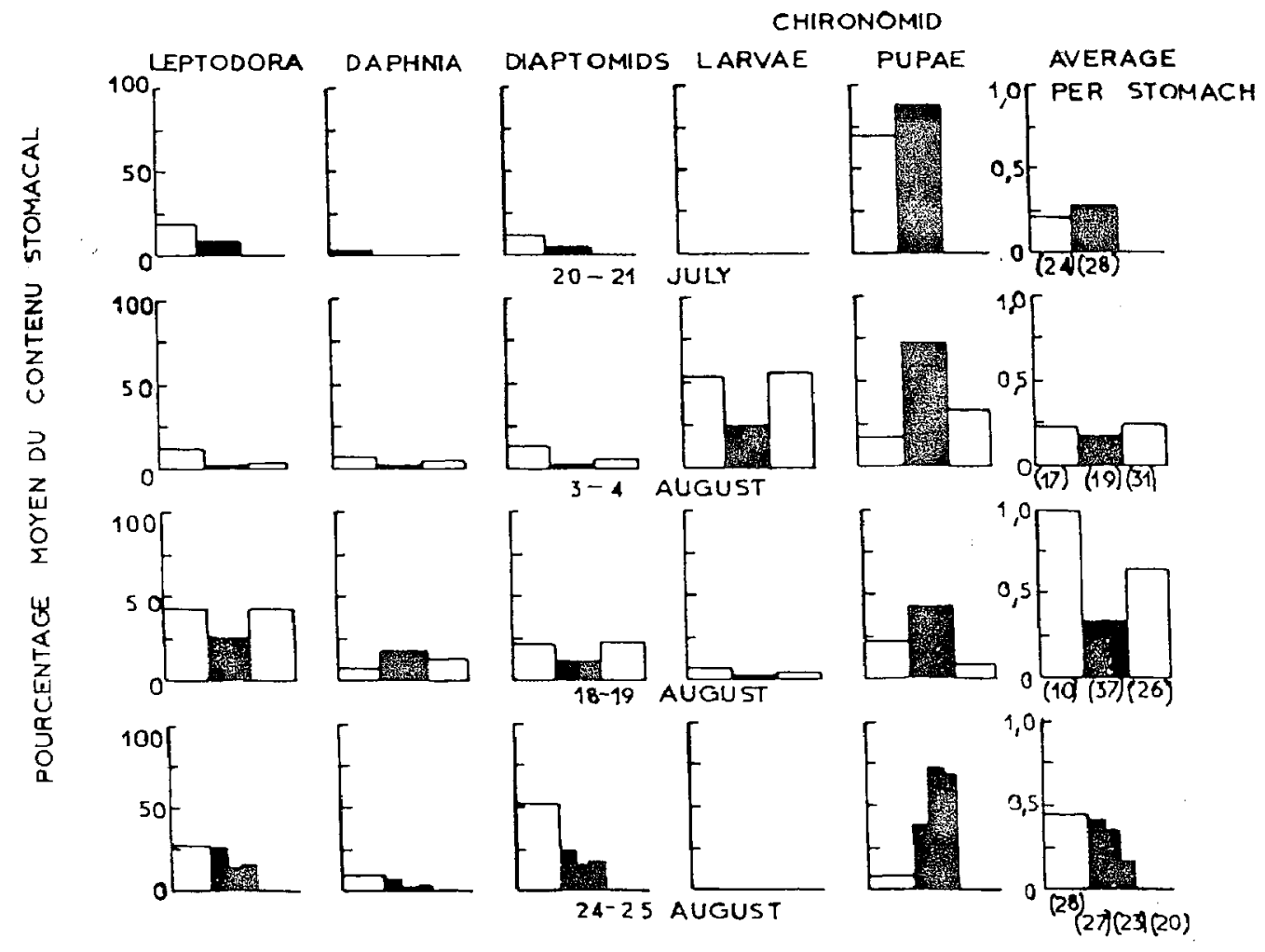

Fig. 8 - Variations nycthémérales du volume et de la composition de la nourriture prise par le Kokanee adulte au cours de l'été 1969.

Les histogrammes blancs correspondent aux poissons capturès le jour, les noirs à ceux capturés la nuit.

Les histogrammes de juillet et de fin d'août représentent les résultats d'une journée et de la nuit suivante. Ceux des 3 et 4 août et des 18 et 19 août portent sur un jour, une nuit et le jour suivant. Le nombre des poissons échantillonnés est donné entre parenthèses.

(D'après NORTHCOTE et LORZ 1966) 

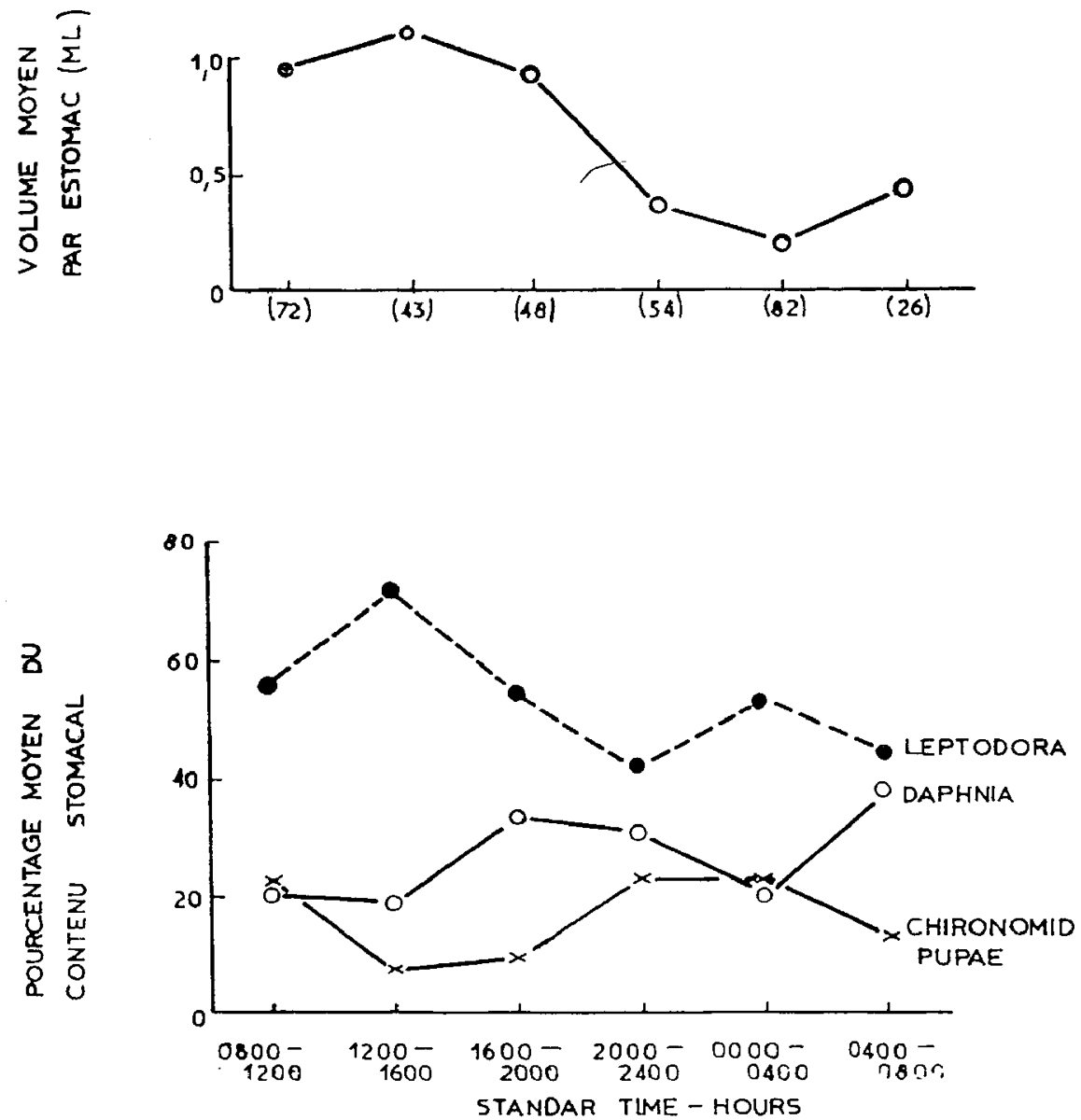

Fig. 9 - Variations nycthémérales du volume et de la composition de la nourriture prise par le Kokanee adulte de Nicola Lake pendant des intervalles de $4 \mathrm{H}$ les 25 et 26 août 1963.

Le nombre des poissons échantillonnés est indiqué entre parenthèses. (D'après NORTHCOTE et LORZ 1966) 
formes crustacées de zooplancton dans la nourriture du Kokanee adulte : Cladocères, tels que Daphnia et Bosmina et Copépodes tels que Epischura, Diaṕtomus ou Cyclops en sont les représentants habituels.

PLATTS avait fait remarquer en 1958 la présence de Leptodora dans les estomacs des Kokanee adultes mais aucune étude précédente n'avait souligné l'importance de la prédation vis-à-vis de ce genre de plancton.

CLEMENS et ses collaborateurs en 1939 avaient considéré les Chironomides comme des composants mineurs de la nourriture du Kokanee adulte.

Au Japon, dans le lac Yunnoko, les larves de Chironomides constituent $12,1 \%$ et les nymphes $15,1 \%$ de la nourriture trouvée lors de l'examen des estomacs de 33 Kokanee (SHIRAISHI et TAKAGI 1955).

En réalité ces pourcentages sont parfois élevés. La présence des Chironomides en quantité non négligeable dans les estomacs des Kokanee de Nicola Lake semble remettre en question la nature de la nourriture telle qu'elle avait été définie antérieurement : le Kokanee était considéré comme un consommateur de plancton exclusivement, non de Benthos. Or bien que le Kokanee de Nicola Lake soit, de jour comme de nuit, assez près du fond (NORTHCOTE 1964) la présence des larves de chironomides dans les estomacs n'indique pas nécessairement qu'il puise sa nourriture sur le fond.

MUNDIE (1959) a trouvé dans le lac La Ronge (Colombie Britanique) une grande variété de larves de Chironomides près de la surface, en des endroits où la profondeur dépassait $40 \mathrm{~m}$ : ceci semble donc confirmer le fait que le Kokanee ne prend pas sa nourriture sur le fond.

\section{VII - COMPETITION INTERSPECIFIQUE}

Le Kokanee, consommateur de zooplancton, se classe dans les consommateurs secondaires (C2) au mème titre que la truite et le Sockeye anadrome. Mais, alors que la truite peut être à la fois $\mathrm{C} 3-\mathrm{C} 4 \ldots$.. le Kokanee a un éventail de possibilités nutritionnelles moins large, bien qu'il lui arrive parfois de consommer quelques alevins.

Dans Cultus Lake où le Kokanee cohabite avec le Sockeye anadrome et les Résiduels issus de ce dernier, il y a parmi eux une compétition alimentaire : il est alors préférable du point de vue économique de favoriser l'extention du Sockeye anadrome plutôt que celle du Kokanee.

Dans les eaux contenant déjà de la truite, la présence d'une population importante de Kokanee réduit la production de la truite, car cette dernière consomme également du zooplanction : ceci est encore plus accentué quand, dans un lac, la principale source de nourriture est constituée essentiellement par le zooplancton.

Le Kokanee est très bien adapté à son régime alimentaire : en effet, les premiers arcs branchiaux possèdent des Branchiospines longues et nombreuses (28 à 40) qui lui permettent de filtrer efficacement le zooplancton. La truite, moins bien adaptée à cause de ses branchiospines plus courtes et moins nombreuses (17 à 21), capture moins efficacement le zooplancton, à tel point que dans les lacs peu riches en zooplancton le Kokanee est un rival certain.

Dans le Vermont où l'on implanta le Kokanee pour la première fois, dans le but de fournir un poisson fourrage aux truites de lacs et aux saumons, et 
non pas dans l'intention d'alimenter la pêche en Kokanee, l'opération fut un réel succès. En effet, le Kokanee servit à la fois de poisson-fourrage et de poisson de pêche. Bien qu'il constitue un poisson-fourrage pour la truite dans certains Etats (Idaho, Colombie Britannique), cela n'a pu être observé en Californie.

En effet le rythme de croissance relativement rapide, les habitudes de concentration pélagique, et la sténothermie du Kokanee font en sorte que les grosses truites s'attaquent plutôt à leurs propres alevins qu'à ceux des Kokanee, d'où l'importante réduction des populations de truites dans les eaux renfermant simultanément la truite et le Kokanee.

Dans les petits lacs, la compétition alimentaire truite-Kokanee est nettement défavorable à la truite, alors que dans les eaux riches en nourriture cela parait moins évident.

Dans le Priest Lake, Idaho, l'introduction du Kokanee dans les eaux à truite conduit à l'élimination presque totale de : Salmo clarkii lewisi. En 1955 la truite ne présentait plus que $6 \%$ de la pêche alors que le Kokanee en constituait les $94 \%$.

En Californie, Salmo trutta et le Kokanee utilisent les zones de fraie des mêmes cours d'eaux. Quand il s'agit du Kokanee émigrant en août-septembre, la truite ne lui dispute pas le terrain, mais quand le Kokanee émigre en novembredécembre il se trouve en même temps que cette dernière sur les zones de ponte et c'est souvent la truite qui supporte les conséquences de cette compétition.

\section{VIII - AGES ET TAILles a maturite}

Le cycle de vie des Kokanee varie de 2 à 7 ans. Tous meurent après la reproduction (Photo 2). Dans leurs contrées d'origine, la durée du cycle est de 4 ans. Dans la Skeena, au nord de la Colombie Britannique, la maturité se situe vers 5 ans, alors que dans le Sud c'est plutôt vers 3 ans à compter de ta fécondation de l'œuf. Quelques mâles précoces arrivent à maturité à 2 ans. Cette variabilité est illustrée dans les différents bassins de Kootenay Lake (fig. 1) : dans les bras Ouest et Sud l'âge moyen des reproducteurs est de 3 ans alors qu'il est de 4 ans pour ceux du bras Nord. Dans le bassin supérieur de Priest Lake (Idaho), le Kokanee implanté arrive à maturité à 5 ans et à 4 ans dans le reste du lac.

A quelques exceptions près, l'âge de maturité augmente avec la latitude, mais aussi avec l'altitude. Cette tendance est bien marquée en Californie et en Colombie Britannique où l'âge de maturité passe de 2 à 5 ans au fur et à mesure que l'on progresse vers le Nord.

Cependant dans bien des cas les informations concernant l'âge de maturité du Kokanee, en Californie, sont faussées car, lors des implantations, on a l'habitude de mélanger les œufs de différentes souches, certaines ne donnant aucun résultat. II arrive que des souches qui mûrissent normalement à 4 ans, mûrissent à 3 ans dans les eaux de Californie. On ignore si cela est dû à une augmentation de la température ou à un ajustement temporaire à une nourriture plus abondante durant les premiers stades de croissance.

Les sources d'œufs variees (WASHINGTON, IDAHO, MONTANA et COLORADO) ont abouti à un mélange de poissons d'origines géographiques différentes. Et effectivement l'âge à maturité varie à l'intérieur de ce mélange. II semble donc qu'il n'y ait pas de croisements entre ces races, lors de la reproduction. 


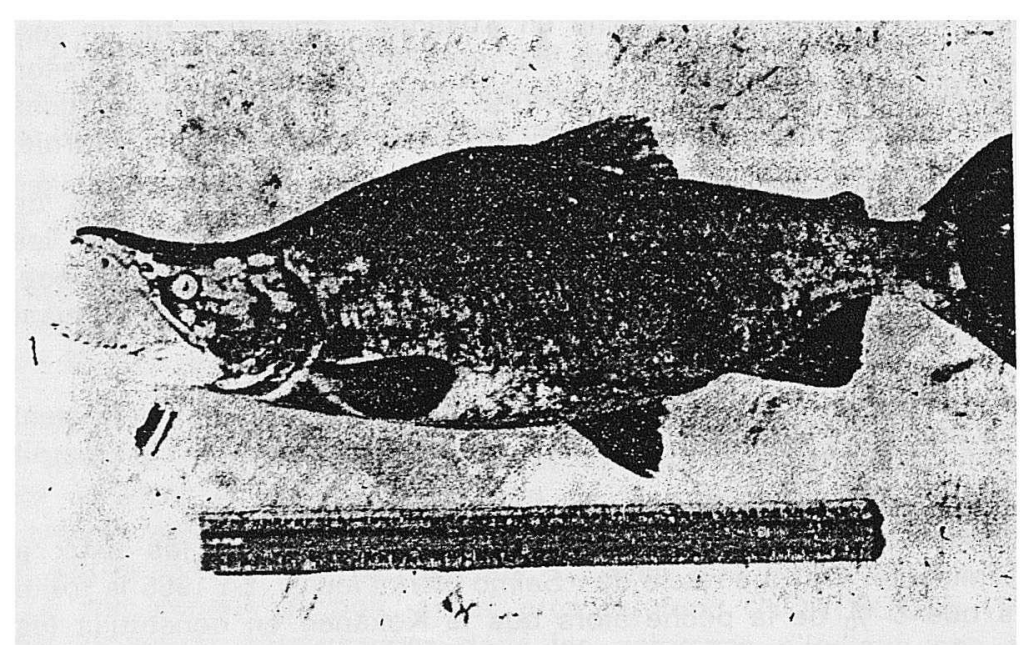

\section{Photo 1}

Kokanee mâle en période de reproduction.

Donner Lake, Californie.

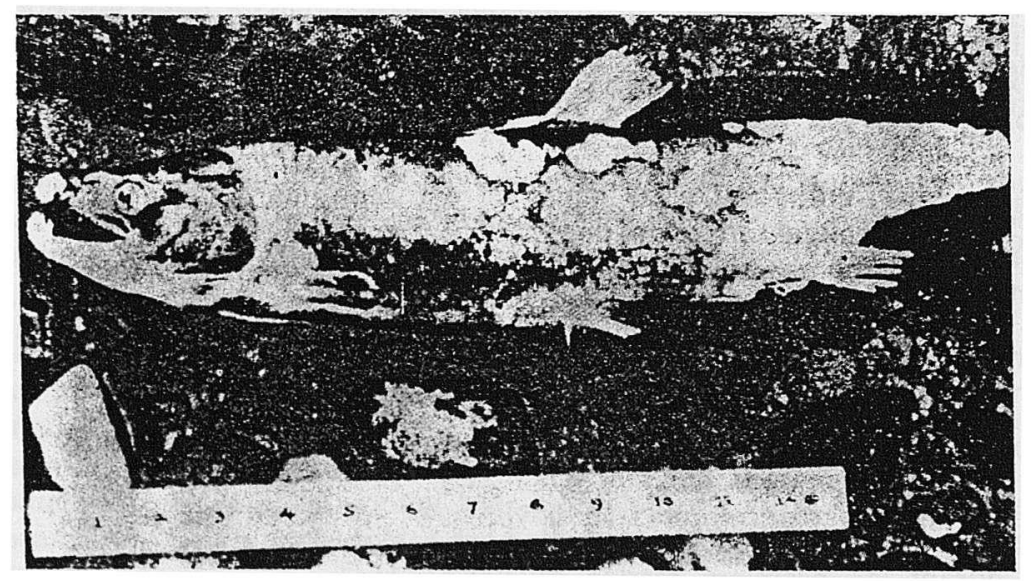

Photo 2

Kokanee mâle trouvé mort après la fraie.

Donner Lake, Californie. 
Dans Cultus Lake en Colombie Britannique les mâles mûrissent généralement en moyenne 1 an avant les femelles. Les mâles Résiduels mûrissent à 2 ou 3 ans, quelquefois à 4 ans et les femelles à 3 ou 4 ans.

Les plus grands poissons se rencontrent gènéralement dans les eaux à faible densité de population de Kokanee comme les grands lacs et réservoirs. Ainsi dans Donner Lake (Californie) la plupart des reproducteurs ont 4 ans, quelques-uns 3 ans. Ce lac, de tailie moyenne, profond et froid, semble ideal pour le Kokanee malgré l'absence d'affluents pour la fraie et les baisses de niveau affectant ses rives, compromettant ainsi l'incubation des œufs. La taille à maturité est voisine de $38 \mathrm{~cm}$ (L. à la Fourche). KIMSEY (1951) a observé des reproducteurs de $45 \mathrm{~cm}$. Mais en septembre 1962, les implantations de 1959 et 1960 ont donné des reproducteurs précoces de 2 ou 3 ans dont la taille, oscillant entre 22 et $40 \mathrm{~cm}$, avait une moyenne de $30 \mathrm{~cm}$ (Photo 1).

Avant les implantations de 1959 et 1960, la taille moyenne des Kokanee de Donner Lake était de $46 \mathrm{~cm}$ pour un poids moyen de 2 livres.

C'est une moyenne qui semble forte par rapport à beaucoup d'autres populations. Ce simple exemple montre la grande variabilité de la taille à maturité à l'intérieur d'un seul lac.

Dans les lacs du Nord de la Colombie Britannique la taille moyenne du Kokanee est de 20 à $23 \mathrm{~cm}$, alors que dans ceux du Sud elle est de 30 à $38 \mathrm{~cm}$.

Dans le Vermont, des œufs provenant de la même source donnent des Kokanee dépassant $52 \mathrm{~cm}$ dans certains lacs, alors que dans d'autres lacs ils ne dépassent jamais $28 \mathrm{~cm}$.

II semble de plus en plus ètabli que les différences de taille à maturité entre les populations landlocked sont dèterminées par les conditions d'environnement.

Généralement la taille moyenne du Kokanee adulte oscille entre 20 et $52 \mathrm{~cm}$. Le record de poids est de 4 livres.

Mais il est très difficile de comparer les rythmes de croissance des Kokanee en raison de l'hétérogénéité des races, des renseignements fragmentaires obtenus et des facteurs externes tels la latitude, l'altitude, la température, l'état trophique des lacs et bien d'autres encore.

\section{IX - REPRODUCTION}

\section{1 - Période de fraie}

La période de fraie varie considérablement suivant les régions, mais surtout selon les races.

Dans le lac Pend Oreille, Idaho, elle se situe d'août à février avec un maximum en novembre et décembre. C'est surtout en novembre qu'elle a lieu dans le Flathead Lake. Montana, et en octobre-novembre dans l'Orégon.

En Californie on distingue deux groupes : le premier qui fraye en aoûtseptembre et début octobre, les adultes émigrant à l'amont des rivières dès la fin juillet, et le second qui fraye de fin octobre à février.

La période de fraie semble être influencée par l'environnement. On a transplanté une souche de Kokanee frayant en août de Chatterdown Creek 
dans Trinity Lake en 1961. En 1962, on n'avait pas encore décelé de signes de fraie dans Trinity Lake, 15 jours après le début de la fraie dans Chatterdown Creek. Toutefois ces données n'ont pas permis de déterminer les composantes de l'environnement qui agissaient en temps que facteurs.

\section{2 - Température de fraie.}

La fraie exige une température relativement basse allant de $5^{\circ} \mathrm{C}$ à $120 \mathrm{C}$.

Dans le lac Granby, au Colorado, le Kokanee commence à pondre quand la température de surface est de $5 \circ \mathrm{C}$

Les reproducteurs d'automne de Shasta lake, en Californie, remontent dans les rivières quand la température descend en-dessous de $14,5 \circ \mathrm{C}$, mais ne commencent à frayer que vers $12,50 \mathrm{C}$. L'échec général des migrations d'automne dans les autres affluents de Shasta Lake est certainement dú à une température trop élevée.

\section{3 - Fécondité}

La fécondité du Kokanee est fonction de la race et des conditions locales. La femelle Kokanee pond de 300 à 1600 œufs suivant sa taille. Les femelles du lac Granby, au Colorado, ont $38 \mathrm{~cm}$ et pondent 1670 œufs. A Salt Springs Reservoir en 1943, 626 femelles de $27,5 \mathrm{~cm}$ à $30 \mathrm{~cm}$ avaient en moyenne 479 œufs chacune (CURTIS et FRASER, 1948).

Des femelles de $24 \mathrm{~cm}$, dans le Nevada, n'avaient en moyenne que 387 œufs. Les nombres d'œufs sont obtenus au cours des opérations de fécondation artificielle et constituent les moyennes des œufs extraits des femelles.

WHITT (1957) estime que $93 \%$ des cufs sont déposés lors de la fraie, le reste demeure dans le poisson mort.

\section{4 - Caractéristiques des frayères}

La fraie a lieu soit en bordure des lacs soit dans leurs petits affluents.

Des expériences ont montré que le Kokanee parvenu à maturité ne dépose pas ses œufs et meurt quand il y a absence totale de courant. Quand la fraie a lieu sur les bordures des lacs, il est presque indispensable que des petites sources sous-lacustres entretiennent un courant permanent sur le fond pour que celle-ci ait des chances d'aboutir.

On suppose que les diverses souches de Kokanee ont des aptitudes plus ou moins marquées à frayer en lac, mais on n'a jamais isolé de souche présentant nettement ce caractère.

DELISLE (1962) estime que la vitesse du courant dans les frayères doit ètre inférieure à $75 \mathrm{~cm} / \mathrm{s}$. Des Kokanee frayant dar.s la rivière issue de Bucks Lake se fixaient dans les endroits où la vitesse de l'eau, mesurée à $6 \mathrm{~cm}$ du fond, était inférieure à $75 \mathrm{~cm} / \mathrm{s}$.

Certains auteurs donnent une vitesse minimale nécessaire de $0,1 \mathrm{~m} / \mathrm{s}$. Cependant il faut noter que ces vitesses de courant, mesurées près du fond, peuvent ou non, suivant le cas, donner une indication sur l'écoulement de l'eau à travers les graviers où se trouvent les œufs. Ceci est essentiellement fonction du degré de perméabilité du gravier. 
Le substrat est constitué de sables, graviers cailloux ou blocs (photo 3 ). Le diamètre des éléments dominant varie de 1 à $10 \mathrm{~cm}$. RICHARD (1954) note que dans Taylor Creeck, affluent du lac Tahoe (Californie), la plupart des activités de fraie sont localisées sur des graviers de moyenne et petite taille, à la fois dans des a pools - et des adiers . Mais dans la partie aval, méandriforme, quelques Kokanee ont frayé dans un sable grossier. RICHARD collecta beaucoup d'œufs dans cette zone sableuse mais n'en trouva aucun vivant.

Quand le lac ne reçoit pas de rivière les Kokanee vont souvent frayer en aval, dans la rivière de sortie. Cela cause souvent de grandes pertes d'alevins qui sont entrainés vers l'aval et meurent. On y remédie en construisant de petits barrages.

Au moment de la fraie, les Kokanee semblent avoir perdu le pouvoir de remonter les rivières de leurs ancêtres; une simple petite chute, facilement franchie par les Sockeye anadromes, stoppe leur mouvement vers l'amont. C'est la femelle qui prépare le terrain pour la réception des œufs. Elle fait des excavations dans les graviers du fond, tandis que le mâle semble surveiller les lieux. Les trous ont une dizaine de centimètres de profondeur. La femelle émet ses œufs en plusieurs fois et progresse vers l'amont si bien que les nids d'aval, alimentés en œufs, sont progressivement recouverts par les graviers soulevés en amont au fur et à mesure que la fraie se poursuit.

Les œufs sont ensevelis sous une couche de gravier de 10 à $18 \mathrm{~cm}$ d'épaisseur. Un courant d'eau ininterrompu et une bonne oxygénation sont les facteurs essentiels. En 1932, KROKHIN et KROGIUS ont mesuré la température, la teneur en oxygène dissous et le $\mathrm{pH}$ de plusieurs zones de ponte. Les résultats sont les suivants :

\begin{tabular}{|l|c|c|c|c|}
\hline Sol de ponte. & $\begin{array}{c}\text { Température. } \\
\text { o C }\end{array}$ & \multicolumn{2}{|c|}{ Oxygène. } & pH. \\
\cline { 2 - 3 } & $\mathbf{m g} / 1$ & $\begin{array}{c}\% \text { de } \\
\text { saturation }\end{array}$ & \\
\hline Bordure de lac. & 4,44 & 11,68 & 89,21 & 7,2 \\
Rivière. & 0,05 & 12,50 & 92,14 & 7,1 \\
Source. & 3,97 & 10,22 & 77,05 & 6,7 \\
\hline
\end{tabular}

\section{5 - Incubation}

Les températures varient de $0 \circ \mathrm{C}$ à $130 \mathrm{C}$ pendant l'incubation. Une température de $3,5^{\circ} \mathrm{C}$ correspond à la limite inférieure à laquelle les œufs peuvent donner des embryons.

A la pisciculture de Hot Creek, Mono County, quelques œufs ont éclos à $130 \mathrm{C}$, mais la mortalité au stade de la vésicule vitelline fut très forte. La période allant de l'éclosion à la première prise de nourriture était de 10 à 12 jours.

Des observations très instructives ont été faites par KIMSEY en 1949, dans le Donner Lake (Californie). Des œufs de Kokanee, déposés dans des nids de gravier à 10 ou $15 \mathrm{~cm}$ de profondeur en bordure de ce lac, ont été surveillés pendant toute la durée de l'incubation. La fraie a commencé au début du mois de novembre. Le 15 et le 29, neuf nids furent repérés et marqués. Les œufs commencèrent à éclore, dans certains de ces nids, vers le 20 janvier (environ 2 mois d'incubation). Ces nids étaient situés dans des zones de sources (température voisine de $70 \mathrm{C}$ à $9 \circ \mathrm{C}$; la température de l'eau du lac se situant 


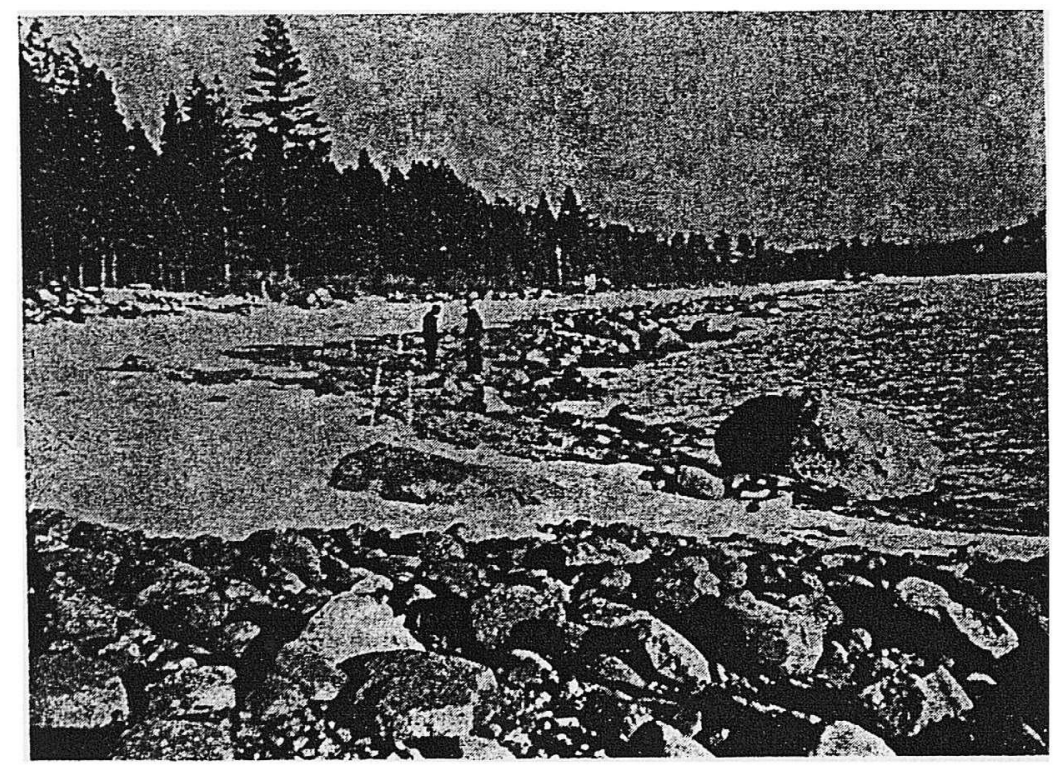

Photo 3

Frayères exposées à l'air et au gel le 30 décembre 1949, en bordure de Donner Lake. Les nids, alors au dessus du niveau de l'eau, gelèrent. Les piquets marquent l'emplacement de certains nids. (D'après KIMSEY, 1949).

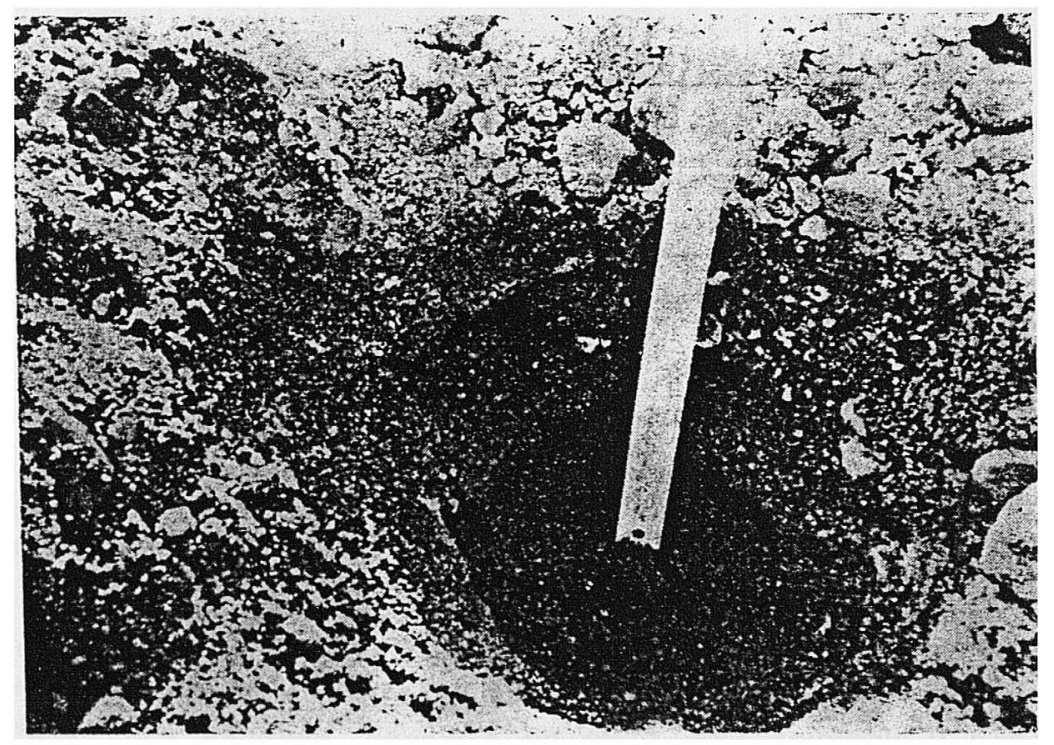

Photo 4

Nid gelé à une profondeur de $17,5 \mathrm{~cm}$, le 30 décembre 1949 . Tous les œufs furent tués par le gel. Donner Lake. (D'après KIMSEY, 1949). 
aux alentours de 5 à $60 \mathrm{C}$ ). De nombreux nids se sont trouvés asséchés par une baisse importante du niveau de l'eau et ont subi un gel prolongé : tous. leurs ceufs furent détruits (Photos 3 et 4 ).

Par contre, d'autres nids qui se sont trouvés au-dessus du niveau d $\epsilon^{\prime}$ l'eau, mais qui sont restés inhibès par capillarité ont donné des cufs qui, malgré un léger gel temporaire, transplantés dans la pisciculture Tahoe, ont éclos le 15 mars (température 6o $\mathrm{C}$ ).

Dans le lac Pend Oreille les ceufs de Kokanee éclosent au bout de 3 à 5 mois suivant la température de l'eau; les alevins conservent leur sac vitellin pendant 30 à 40 jours puis sortent du gravier et émigrent dans le lac où ils demeurent jusqu'à leur maturité. (JEPPSON 1955).

\section{CONCLUSION}

L'introduction du Kokanee dans un milieu lacustre donné peut avoir des résultats variables et, dans ce domaine, il est très difficile de faire des pronostics sûrs.

Dans de nombreux cas les implantations furent des échecs. On n'en connait pas toujours, d'après les auteurs, les raisons exactes. Cependant la sensibilité du Kokanee à une température trop élevée exige un hypolimnion présentant toutes les conditiors recuises de fraicheur et d'oxygénation. Ceci est fondamental. Les frayères ont des caractéristiques trés strictes, comme nous l'avons vu; aussi les possibilités d'extension exagérée sont-elles très faibles.

Poisson de sport combatif, il est d'autant plus apprécié que les techniques de pêche évoluent et que les pêcheurs connaissent mieux ses habitudes. C'est un Salmonidé dont la chair a la réputation d'être excellente.

$\mathrm{Au}$ Japon, on a longtemps prêté une sérieuse attention à ce poisson et de nombreux efforts ont été faits pour la conservation et l'accroissement de l'espèce, objet favori de la pêche sportive et, à un degré moindre, de la pêche commerciale. Bien que la reproduction naturelle assure le maintien de l'espèce dans la plupart des lacs, on pratique la fécondation artificielle en pisciculture. L'incubation en eau de source de 8 à $9 \circ \mathrm{C}$ dure un mois et demi à deux mois (début novembre au 15 décembre). Les alevins sont élevés dans des bassins pendant six mois; on complete leur nourriture par des larves hachées de Mysis et de Diptères.

Quand ils ont atteint 8 à $10 \mathrm{~cm}$, les saumoneaux sont transférés dans les lacs, où ils parviendront à des tailles allant de $25 \mathrm{~cm}$ à $40 \mathrm{~cm}$ suivant les lieux : l'âge et la taille de maturité étant essentiellement fonction du milieu.

En France, est-il souhaitable de porter nos efforts sur la protection de notre Saumon par une pisciculture appropriée ou est-il préférable d'introduire un Saumon d'eau douce? En réalité ces deux mesures ne sont pas opposées mais complémentaires si l'on désire conserver du saumon dans nos eaux continentales: les Suédois l'ont bien compris, qui produisent des smolts en pisciculture et ont adopté avec succès le Kokanee. Le revenu de leurs pêcheries est beaucoup plus important que le coût de l'élevage du poisson.

L'introduction du Kokanee en France peut se révèler intèressante pour certains de nos lacs, à condition d'effectuer une discrimination soigneuse quant à l'origine des œufs et de mettre en place une expérimentation rigoureuse préalable, compte tenu des réserves qui ont été faites au sujet de la compétition interspécifique. 


\section{B I BLIOGRAPHIE}

ALLISON D., 1958 - Age and growth characteristics of Lake Pend Oreille Kokanee, 1957. Idaho Dept. Fish Game Ann. Proj. Rept., Proj. F3-R-, 97.

BELAND D., 1961. - The Lake Arrowhead, San Bernardino County Kokanee Salmon fishery 1957-1960. Fish and Game, Inland Fish. Admin. Rept. No $61-7,14$ p. Miméo.

BRETT I.R., HOLLAND M., ALDERDICE D.F., 1958 - The effect of temperature ont the Cruising Speed of Young Sockeye and Coho Salmon. J. Fish. Res. Bd. Canada 15, 4, $587-605$.

BRUNSON R. B., CASTLE G. B., PIRLE R., 1952 - Studies of Sockeye Salmon (Oncorhynchus nerka) from Flathead Lake, Montana. Proc. Montana Acad. Sci., 12, $35-43$.

CHAPMAN D.W., FORTUNE J.D., 1963 - Ecology of kokanee saimon. Oregon State Game Comm., Res. Div. Rep., $11-42$.

CLEMENS W. A., RAWSON D.S., MC HUGH J. L. 1939 - A biological survey of Okanagan Lake, British Columbia. Bull. Fish. Res. Bd. Canada, No 56, $70 \mathrm{p}$

CURTIS B., FRASER 1.C. 1948 - Kokanee in California. Calif. Fish. and Game. 34, 3, $111-114$.

DELISLE G. B., 1962 - Water velocities tolerated by spanning Kokanee Salmon. Calif. Fish and Game, 48, 1, $77-78$.

FRASER J.C., POLLIT A.F., 1951 - The introduction of Kokanee red salmon (Onchorhynchus nerka kennerlyi) into Lake Tahoe, California, and Nevada. Calif. Fish, and game, 37, 2.

FOERSTER R.E., 1947 - Expermient to deveop sea-run fiom land-loked sockeye salmon (Oncorhynchus nerka kennerlyi). J. Fish Res. Bd. Can., 7, 2, 88 - 93.

HORAK D. L., TANNER H. A., 1964 - The use of vertical gill nets in studying fish depth distribution, Horsetooth Reservoir, Colorado. Trans. Am. Fish. Soc. 93 (2), $137-145$.

JEPPSON P., 1955 - Evaluation of spawning areas in Lake Pend Oreille and tributaries upstream from Alberni Falls Dam in Idaho, April 1, 1954Mai 31, 1955, including supplemental information on the life history of the Kokanee. Idaho. dept. Fish and Game, D J Federal Aid Proj. F-3R-4. and F3-R-5 (Work Plan 2) Ann. Sum. Dept.

JOHNSON W.E., 1956 - On the distribution of young sockeye salmon (Oncorhynchus nerka) in Babine and Nilkitkwa Lakes, B.C.J. Fish. Res. Can., $13,695-708$.

JOHNSON W.E., 1958 - Density and distribution of young sockeye salmon (O. Nerka) troughout a multibassin lake system. J. Fish. Res. Bd. Can 13, $695-708$.

JOHNSON W.E., 1961 - Aspects of the ecology of a pelagic zooplanctoneating fish. Verh. Internat. Verein. Limnol., 14, 727 - 731.

JORDAN D.S., 1925 - Fishes. D. Appleton and Co. 773 pp.

KIMSEY J.B., 1951 - Notes on kokanee spawning in Donner Lake, California 1949. Calif. Fish and Game, 37, 3, 273-279.

KROKHIN E.M., 1963 - The propragation and utilization of kokanee salmon in Japan. Rybn. Khoz. 39 (11), 89-91.

LEGENDRE V., 1966 - Le Saumon d'eau douce du Québec : le poisson de sport de l'avenir. Tourism, Fish and Game vol. 1 nos 12 à 15, vol. 2 nos 1 et 2. 
LORZ H.W., NORTHCOTE T.G., 1965 - Factors affecting stream location, and timing and intensity of entry by spawning kokanee (Oncorhynchus nerka) into an inlet of Nicola Lake, British Columbia. J. Fish. Res. Bd. Canada. 22 (3), 665-687.

Mc. MAHON V.H., 1954 - The abudance and Distribution of Entomostracan Plankton at Lakelse B.C., 1949 - 1952. J. Fish. Res. Bd. Canada. II, 4, 1964.

MUNDIE J.H., 1959 - The diurnal activity of the larger invertebrates at the surface of Lake la Ronge, Saskatchevan. Canadian J. Zool. 37, 945-956.

NORTHCOTE T.G., LORZ H.W., MACLEOD J.C., 1964 - Studies on diel vertical movement of fishes in a British Columbia lake. Verh. Int. Verein. Limnol., 15, 940-946.

NORTHCOTE T.G., LORZ H.W., 1966 - Seasonal and diel changes in food of adult Kokanee (Oncorhynchus nerka) in Nicola Lake, British Columbia.

J. Fish. Res. Bd. Canada, 23, (8), 1259-1263.

PLATTS W.G., 1958 - A comparison of limnological data collected from Lake Pend Oreille during and after dam construction with food habits of the kokanee. Idaho State Dept. Fish Game, Prog. Rep., p. 73.

RICHARD, J.B., 1954 - Observations of Kokanee salmon spawning in the tributaries of Lake Tahoe, October 1952 to February 1953. Cal. Dept. Fish and Game, Inland Fish. Admin. Rept. No 54-3, 14 pp. (typerwritten).

RICHARD 1., HALLOCK and DONALD H., FRY I.R., 1967 - Five species of Salmon Oncorhynchus, in the Sacremento River, California. Cal. Fish. and Game, 53 (1), 5-22.

RICKER W.E., 1937 - The food and the supply of Sockeye salmon (Oncorhynchus nerka Walbaum) in Cultus Lake, British Columbia. J. Biol. Bd. Canada, 3 (5), $450-468$.

RICKER W.E., 1940 - On the origin of kokanee, a fresh-water type of sockeye salmon. Trans. Roy. Soc. Canada, Sect. 5, $121-135$.

RICKER W. E., 1938 - "Residual" and Kokanee, salmon in Cultus Lake. Fish Res. bd. Canada 4, 3, 192 - 218.

RICKER W.E., 1959 - Additional Observations Concerning Residual Sockeye and Kokanee (Oncorhynchus nerka). J. Fish. Res. Bd. Canada 16, 6, 897-902.

SEELEY C.M., Mc. CAMMON G.W., 1966 - Kokanee. Dept. Fish and Game, Inland Fisheries Management, 274-294.

SHIRAISHI Y., TAKAGI M., 1955 - Food habit of trout in Lake Yunoko, with reference to the morphology of boby and digestive organs. Bull. Fresh. Fish. Res. Lab., 5, 1, $11-30$.

SPILLMANN Ch. J., 1961 - Faune de France, 65 Poissons d'eau douce, 32-64.

VERNON E.H., 1957 - Morphometric comparaison of three races of Kokanee (Oncorhynchus nerka). Within a large British Columbia Lake. J. Fish. Res. Bd. Canada, 14, 4, 573-598.

VIBERT R., 1950 - Recherches sur le Saumon de l'Adour. Ann. Stat. Cent. Hydrobiol. Appl. t 3, 27-149.

WALLIS O.L., Bond C.E., 1950 - Establishment of kokanee in crater Lake, Oregon. Jour. Wildl. Mgt., 14, 2, $190-193$.

WISBY W.J., HASLER A.D., 1954. Effect of olfactory occlusion on migrating silver salmon (O. kisutch). J. Fish. Res. Bd. Canada, 11, 4, $472-478$.

WHITT C.R., 1957 - Evaluation of spawning areas in Lake Pend-Oreille and. tributaries upstream from Alberni Falls Dam in Idaho, June 1, 1956 to may 31, 1957. Idaho Dept. Fish and Game, D-J Federal Aid Projs. F3-R-6 and F-3-R-7 (Work Plan 2), Ann. Summ. Rept., 20 pp. (Mimeo.). 